\title{
Uso de um esquema implícito e de splines para a simulação numérica do processo de resfriamento de frutas esféricas
}

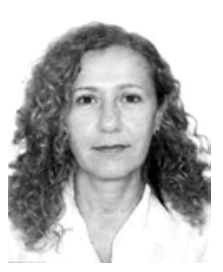

Mariângela Amendola ${ }^{1}$ \& Bárbara Teruel ${ }^{2}$

1 FEAGRI/UNICAMP. Fone: (19) 3788-1065, Fax: (19) 3788-1010, Caixa Postal 6011, CEP. 13083-970, Campinas, SP. E-mail: amendola@agr.unicamp.br (Foto)

2 FEAGRI/UNICAMP. Fone: (19) 37881004, E-mail: barbarat@agr.unicamp.br

Protocolo 185 - 29/11/2002 - Aprovado em 5/4/2004

\begin{abstract}
Resumo: Este trabalho teve como objetivo desenvolver um estudo teórico através do modelo matemático de Fourier, em sua forma unidimensional e em coordenadas esféricas, o que é descrito por uma equação diferencial parcial linear de segunda ordem, para a obtenção das curvas e do tempo de resfriamento de frutas esféricas. Utilizou-se o Método das Diferenças Finitas (MDF) segundo o esquema implícito no ambiente computacional Matlab 6.1, analisando-se e comparando os principais resultados através do método de ajuste spline. 0 modelo matemático foi validado com dados experimentais do resfriamento de laranja Valência num sistema com ar forçado $(\mathrm{Q}=$ $1933 \mathrm{~m}^{3} \mathrm{~h}^{-1}$, e velocidade em torno de $1 \mathrm{~m} \mathrm{~s}^{-1}$ ), com temperatura inicial de $26,57^{\circ} \mathrm{C}$ e resfriada até $1^{\circ} \mathrm{C}$. Os resultados teóricos obtidos com a simulação foram comparados com os encontrados com a aplicação de um modelo bidimensional desenvolvido em coordenadas esferoidais prolato, com o emprego do Método dos Volumes Finitos (MVF) implementado em Fortran 77. Os dois modelos e métodos aplicados mostram-se aptos para caracterizar o processo de resfriamento das frutas, principalmente em termos de valores práticos específicos, uma vez que geraram resíduos desprezíveis. Apesar destes resultados, o valor do coeficiente convectivo de transferência de calor foi significativamente diferente; tal variação mostra como este coeficiente é sensível a vários fatores do processo de resfriamento e, ainda, que se deve aprofundar as pesquisas para a determinação efetiva do valor do mesmo.
\end{abstract}

Palavras-chave: laranjas Valencia, tempo de resfriamento, coeficiente convectivo de transferência de calor

\section{Use of an implicit scheme and splines for the numerical simulation of the cooling process of spherical fruits}

\begin{abstract}
The objective of this research was to conduct a study using the Fourier one-dimensional mathematical model, in spherical coordinates, which is described as a second order linear partial differential equation, in order to know the curves and the cooling time of spherical fruits. The implicit Finite Differences Method (FDM) implemented in the computational environment Matlab 6.1 was used, analysing and comparing the main results by the spline method. The validation of the mathematical model was carried out using experimental data of Valencia oranges inside a system with forced air $\left(\mathrm{Q}=1933 \mathrm{~m}^{3} \mathrm{~h}^{-1}\right.$ and air velocity around $\left.1 \mathrm{~m} \mathrm{~s}^{-1}\right)$, at the initial temperature of $26.57^{\circ} \mathrm{C}$ and cooled till $1{ }^{\circ} \mathrm{C}$. The theoretical results obtained in this way were compared with those obtained from the use of a two dimensional mathematical model, in spheroid prolato coordinates, using the Finite Volume Method (FVM) implemented in Fortran 77 code. Both of these model and methods were able to characterize the cooling process, mainly in terms of the practical specific values, once the residues can be disregarded. Although, the value of the convective heat transfer coefficient was significantly different; this variation shows how this coefficient is sensible to the assumptions of the cooling process and that this research must continue in order to determine its effective value.
\end{abstract}

Key words: oranges Valencia, cooling time, convective heat transfer coefficient 


\section{INTRODUÇÃO}

O resfriamento de produtos agrícolas, dentre eles as frutas, é um processo de transferência de calor, no qual estão envolvidos, basicamente, dois dos mecanismos de transferência de calor: a condução e a convecção. Em particular, o problema da predição do tempo de resfriamento dos produtos e a caracterização dos parâmetros que caracterizam o processo de transferência de calor durante o resfriamento, começaram a ser estudados por Smith \& Bennett (1965).

Um dos grandes desafios continua sendo a determinação do coeficiente convectivo de transferência de calor $\left(h_{c}\right)$ o qual tem papel determinante nos processos que envolvem convecção. Uma das causas mais comuns de erro no cálculo da temperatura dos produtos é originada pelo valor adotado para este coeficiente, ou pelo valor obtido aplicando-se algum dos métodos matemáticos conhecidos.

A determinação deste coeficiente, usando-se métodos experimentais, é muito complexa pela quantidade de fatores que o influenciam. Soma-se o fato de que a determinação experimental da temperatura da superfície implica em um grau de incerteza grande, pela dificuldade para a colocação e fixação do sensor na superfície das frutas ( Amendola, 2003).

$\mathrm{Na}$ maioria dos modelos descritos na literatura, o valor deste coeficiente é adotado seguindo-se algumas faixas recomendadas pela literatura, as quais nem sempre caracterizam, de forma adequada, o processo em particular (Benavides \& Avendaño, 1997).

Para o estudo teórico de processos de resfriamento de produtos agrícolas vários desses modelos apresentam equações que são resolvidas aplicando-se métodos analíticos e/ou numéricos, dependendo de sua complexidade, geometria, tipos de simplificações físicas adotadas e estabelecimento de condições de contorno (Hayakawa, 1978; Cleland \& Earle, 1982; Hayakawa \& Succar, 1982; Chau et al., 1985; Fraser \& Otten, 1992; Dincer, 1995; Trelea et al., 1998; Amendola, 2004).

Neste sentido, objetivou-se com este trabalho, desenvolver um estudo teórico através da Equação de Difusão de Calor de Fourier, em sua forma unidimensional, descrito por uma equação diferencial parcial linear de segunda ordem, para se obter a curva de resfriamento simulada e o valor do coeficiente convectivo de transferência de calor ajustado, por meio da implementação computacional do esquema implícito do Método das Diferenças Finitas (MDF) e o uso do Método dos Mínimos Quadrados (MMQ) respectivamente, bem como valores de interesse prático pelo uso de spline. O modelo foi validado utilizando-se dados experimentais do resfriamento com ar forçado de laranjas variedade Valência. Os resultados foram comparados com os valores obtidos da simulação do processo pelo uso de um modelo bidimensional desenvolvido em coordenadas esferoidais prolato cuja equação foi resolvida pelo Método dos Volumes Finitos implementado em Fortran 77 (Teruel et al., 2001).

\section{MATERIAL E MÉTODOS}

\section{Dados experimentais}

Os valores de temperatura usados para validação do modelo foram obtidos experimentalmente em pesquisas precedentes
(Teruel et al., 2001). As frutas foram resfriadas num sistema com ar forçado (fluxo de ar, $\mathrm{Q}=1933 \mathrm{~m}^{3} \mathrm{~h}^{-1}$ ) e utilizadas laranjas variedade Valência, as quais foram acondicionadas em caixas plásticas com aproximadamente $40 \%$ de área de abertura efetiva para a passagem do ar, que tinha velocidade de aproximadamente $1 \mathrm{~m} \mathrm{~s}^{-1}$. As leituras de temperatura foram feitas inserindo-se sensores (termopares tipo $\mathrm{T}$, calibrados previamente) que, por sua vez, foram conectados a um sistema de aquisição de dados por computador.

O tempo de resfriamento foi determinado a partir dos dados experimentais de temperatura aproximadamente no centro das frutas, calculando-se a Taxa Adimensional de Temperatura, (TAT) ou de resfriamento. Os tempos de meio e de sete-oitavos do resfriamento foram calculados usando-se a Eq. 1 (Mohsenin, 1980; ASHRAE, 1994):

$$
\mathrm{TAT}_{1 / 2}=\frac{\mathrm{T}_{\mathrm{p}}-\mathrm{T}_{\mathrm{a}}}{\mathrm{T}_{\mathrm{i}}-\mathrm{T}_{\mathrm{a}}}=0,5 \quad \mathrm{TAT}_{7 / 8}=\frac{\mathrm{T}_{\mathrm{p}}-\mathrm{T}_{\mathrm{a}}}{\mathrm{T}_{\mathrm{i}}-\mathrm{T}_{\mathrm{a}}}=0,125
$$

donde $\mathrm{T}_{\mathrm{p}}$ é a temperatura aproximadamente no centro das frutas, Ta é a temperatura do ar resfriado e $T_{i}$ é a temperatura inicial das frutas.

Quando o valor de TAT for de 0,5 , as frutas terão atingido o tempo de meio resfriamento (half cooling time), que nada mais é que o tempo necessário para que a temperatura da fruta $\left(T_{p}\right)$ seja a metade da diferença entre a temperatura inicial das frutas $\left(\mathrm{T}_{\mathrm{i}}\right)$ e a temperatura do ar de resfriamento $\left(\mathrm{T}_{\mathrm{a}}\right)$. Analogamente, quando a Eq. 1 atingir o valor de 0,125, as frutas terão alcançado o tempo de sete-oitavos do resfriamento.

A temperatura média inicial das frutas foi de $26,57^{\circ} \mathrm{C}\left(\mathrm{T}_{\mathrm{i}}\right)$, a temperatura do ar de resfriamento $\left(\mathrm{T}_{\mathrm{a}}\right)$ de $1{ }^{\circ} \mathrm{C}$, e a umidade relativa da câmara de $88,4 \pm 2,0 \%$. Para essas condições experimentais, o meio tempo de resfriamento foi atingido quando a temperatura foi da ordem de $13{ }^{\circ} \mathrm{C}$; enquanto os sete oitavos do tempo de resfriamento quando a temperatura foram de aproximadamente $3{ }^{\circ} \mathrm{C}$. Os valores de TAT foram calculados a partir dos valores de temperatura obtidos experimentalmente.

\section{Modelo matemático e método numérico}

O modelo matemático foi desenvolvido com base na Lei de Fourier que, após sofrer algumas simplificações, foi escrito em coordenadas esféricas, como relatado por Castro \& Amendola (1999) e resultou na equação para determinação da temperatura $\mathrm{T}=\mathrm{T}(\mathrm{r}, \mathrm{t})$ de frutas esféricas:

$$
\frac{\partial T}{\partial t}(r, t)=\alpha\left(\frac{2}{r} \frac{\partial T}{\partial r}(r, t)+\frac{\partial^{2} T}{\partial r^{2}}(r, t)\right) ; \quad t \geq 0, \quad r \in[R, 0]
$$

em que:

$\alpha$ - é a difusividade térmica da fruta $\left(\alpha=\frac{\mathrm{k}_{\mathrm{p}}}{\rho \mathrm{C}_{\mathrm{p}}}\right)$

$\mathrm{k}_{\mathrm{p}}$ - condutividade térmica da fruta, $\mathrm{W} \mathrm{m}^{-1} \mathrm{~K}^{-1}$

$\mathrm{C}_{\mathrm{p}}$ - calor específico da fruta, $\mathrm{J} \mathrm{kg}^{-1} \mathrm{~K}^{-1}$

$\rho$ - densidade da fruta, $\mathrm{kg} \mathrm{m}^{-3}$

$\mathrm{r}$ - coordenada esférica radial, $\mathrm{m}$ 
$\mathrm{R}$ - raio da fruta, $\mathrm{m}$

$\mathrm{T}$ - temperatura no interior da fruta, $\mathrm{K}$

t- tempo, s

A condição inicial associada é dada por:

$$
\mathrm{T}(\mathrm{r}, 0)=\mathrm{T}_{0} ; \quad \mathrm{r} \in[\mathrm{R}, 0]
$$

caso em que se considera que a temperatura inicial $\mathrm{T}$ é constante e igual a $\mathrm{T}_{0}$ em qualquer ponto $\mathrm{r}$ no interior da fruta.

Associada à condição de simetria do problema, tem-se a condição de contorno:

$$
\frac{\partial \mathrm{T}}{\partial \mathrm{r}}(0, \mathrm{t})=0 ; \mathrm{t} \geq 0
$$

Por outro lado, o fluxo de calor $\phi \phi$ que sai através da superfície da fruta, é:

$$
-\mathrm{kp} \frac{\partial \mathrm{T}}{\partial \mathrm{r}}(\mathrm{R}, \mathrm{t})=\phi(\mathrm{t}) ; \quad \mathrm{t} \geq 0
$$

em que:

$$
\phi=\mathrm{h}_{\mathrm{c}}\left[\mathrm{T}_{\mathrm{s}}(\mathrm{t})-\mathrm{T}_{\mathrm{a}}(\mathrm{t})\right]+\mathrm{L}_{\mathrm{v}} \beta\left[\mathrm{p}_{\mathrm{s}}\left(\mathrm{T}_{\mathrm{s}}(\mathrm{t})\right)-\mathrm{p}_{\mathrm{a}}\left(\mathrm{T}_{\mathrm{a}}(\mathrm{t})\right)\right]
$$

donde:

$\phi$ - fluxo de calor através da superfície da fruta, $\mathrm{W} \mathrm{m}^{-2}$

$\mathrm{h}_{\mathrm{c}}$ - coeficiente convectivo de transferência de calor da fruta, $\mathrm{W} \mathrm{m} \mathrm{m}^{-2} \mathrm{~K}^{-1}$

$\beta$ - coeficiente de transferência de massa da fruta, $\mathrm{m}^{-1} \mathrm{~s}$

$\mathrm{T}_{\mathrm{s}}$ - temperatura na superfície da fruta, $\mathrm{K}$

$\mathrm{T}_{\mathrm{a}}$-temperatura na câmara de resfriamento, $\mathrm{K}$

$\mathrm{p}_{\mathrm{a}}$ - pressão parcial de vapor do ar a temperatura $\mathrm{T}_{\mathrm{a}}, \mathrm{Pa}$

$\mathrm{p}_{\mathrm{s}}$ - pressão parcial de vapor na superfície da fruta à temperatura $\mathrm{T}_{\mathrm{s}}, \mathrm{Pa}$

$\mathrm{L}_{v}$ - calor de vaporização da água, $\mathrm{J} \mathrm{kg}^{-1}$

O fluxo de calor está relacionado ao coeficiente de transferência de calor e massa; no entanto, ao se desconsiderar o coeficiente de transferência de massa por ser muito pequeno, estabelece-se outra condição de contorno:

$$
-\mathrm{k}_{\mathrm{p}} \frac{\partial \mathrm{T}}{\partial \mathrm{r}}(\mathrm{R}, \mathrm{t})=\mathrm{h}_{\mathrm{c}}\left[\mathrm{T}_{\mathrm{s}}(\mathrm{t})-\mathrm{T}_{\mathrm{a}}(\mathrm{t})\right] ; \mathrm{t} \geq 0
$$

donde:

$\mathrm{k}_{\mathrm{p}}$ - condutividade térmica da fruta, $\mathrm{W} \mathrm{m} \mathrm{m}^{-1} \mathrm{~K}^{-1}$

$\mathrm{R}$ - raio da fruta, $\mathrm{m}$

As equações foram resolvidas aplicando-se o Método de Diferenças Finitas (MDF) segundo o esquema implícito (Richtmyer \& Morton,1967; Incropera \& Witt, 1990; Ruggiero \& Lopes, 1996).

De acordo com a convenção $\mathrm{T}_{\mathrm{i}}^{\mathrm{n}}=\mathrm{T}(\mathrm{i} \Delta, \mathrm{n} \Delta \mathrm{t})$, para $\mathrm{i}=1, \ldots \mathrm{Nx}$ e $\mathrm{n}=1, \ldots \mathrm{Nt}$; em que $\mathrm{Nx}$ é número de pontos de discretização espacial ao longo da fruta e Nt é o número de integrações no tempo, consideraram-se as aproximações das diferenciais que aparecem nas Eqs. (2), (4) e (7), por diferenças finitas avançadas no tempo e centradas e avançadas no espaço, respectivamente:

$$
\begin{gathered}
\left(\frac{\partial T}{\partial t}(r, t)\right)_{i}^{n} \cong\left(\frac{T_{i}^{n+1}-T_{i}^{n}}{\Delta t}\right) ; O(\Delta \mathrm{t}) \\
\left(\frac{\partial^{2} T}{\partial r^{2}}(r, t)\right)_{i}^{n+1} \cong\left(\frac{T_{i-1}^{n+1}-T_{i}^{n+1}+T_{i+1}^{n+1}}{\Delta r^{2}}\right) ; O\left(\Delta r^{2}\right) \\
\left(\frac{\partial T}{\partial r}(r, t)\right)_{i}^{n+1} \cong \frac{1}{\mathrm{i} \Delta \mathrm{r}}\left(\frac{T_{i+1}^{n+1}-T_{i}^{n+1}}{\Delta r}\right) ; O(\Delta r)
\end{gathered}
$$

Uma vez estabelecida a discretização e escolhidos os valores de $\Delta \mathrm{r}$ e $\Delta \mathrm{t}$, variando os valores de $\mathrm{n}$ e i, obtém-se o sistema de equações algébricas lineares que, quando resolvidas, determinam os valores de $\mathrm{T}_{\mathrm{i}}{ }^{\mathrm{n}}=\mathrm{T}(\mathrm{i} \Delta, \mathrm{n} \Delta \mathrm{t})$, em qualquer $\mathrm{i} \Delta \mathrm{r}$ no interior da fruta e em qualquer $\mathrm{n} \Delta \mathrm{t}$ positivo.

Por meio da análise da influência do número de pontos da malha espacial (tamanho de $\Delta \mathrm{r}$ ), este método garante resultados representativos do modelo matemático.

Após a determinação da malha espacial adequada, a determinação do coeficiente convectivo de transferência de calor foi feita pela seleção da curva de resfriamento simulada que melhor se ajusta à curva de resfriamento experimental, no sentido de se ter o menor resíduo, o que pode ser feito também pelo uso de curvas exponenciais, de polinômios de terceiro e quarto graus ou através de splines cúbicas (Cunha, 2000). Este último método, devido à sua complexidade, foi usado somente para a determinação exata dos valores de meio tempo e sete oitavos de resfriamento experimental, uma vez que apresenta resíduo nulo. Para o cálculo dos resíduos aplicou-se o Método dos Mínimos Quadrados (Boldrini et al., 1980):

$$
\text { ERQ }=\sqrt{\sum_{i=1}^{n}\left(T_{i, \text { num }}^{*}-T_{i, \exp }^{*}\right)^{2}}
$$

que é expresso pela raiz quadrada da soma dos quadrados dos desvios que existem entre os resultados simulados e os dados experimentais. Todos esses procedimentos foram implementados no Matlab 6.1 (Amendola \& Teruel, 2002)

O coeficiente de difusividade térmica usado para a validação do modelo foi de 1,06.10-7 $\mathrm{m}^{2} \mathrm{~s}^{-1}$ (Ramos et al., 1993). Considerou-se uma média do valor da condutividade térmica da casca e do suco, recomendada como $0,5 \mathrm{~W} \mathrm{~m}^{-1}{ }^{\circ} \mathrm{C}^{-1}$ (Trelea et al., 1998).

\section{RESULTADOS E DISCUSSÃO}

No processo de investigação do tamanho da malha, fixouse um valor arbitrário para $h_{c}$ e realizada uma busca do número de elementos, $\mathrm{Nx}$, segundo a estratégia de tomar para $\mathrm{i}=1,2, \ldots$ o valor de $\mathrm{Nx}(\mathrm{i})=2^{\mathrm{cn}(\mathrm{i})}$, em que $\mathrm{cn}(\mathrm{i})=3,4, \ldots$ Isto foi feito até o número i a partir do qual não se observa mais influência na 
curva simulada, pelo método de diferenças finitas (MDF), tomando-se sempre o ponto ccn $(\mathrm{i})=2^{\mathrm{i}}$, na mesma distância do centro da fruta. Na Figura 1 encontram-se os resultados assim obtidos, dentre os quais se verifica que o melhor valor de Nx é 128 ( $\mathrm{cn}=7$ ), e o vetor indica o sentido das simulações obtidas em função do aumento do valor de Nx.

Em função dos resultados anteriores, manteve-se, em todas as simulações que seguem, $\mathrm{Nx}=128$.

Foram realizadas várias simulações pelo MDF para determinação do coeficiente convectivo de transferência de calor $\left(h_{c}\right)$ tomando-se em um primeiro momento, valores intermediários a partir de 20 e até 100, de 10 em 10, como mostrados na Figura 2, na qual se mostram linhas contínuas, apenas para melhor visualização dos resultados, enquanto o vetor indica o sentido das simulações para maiores valores de $h_{c}$.

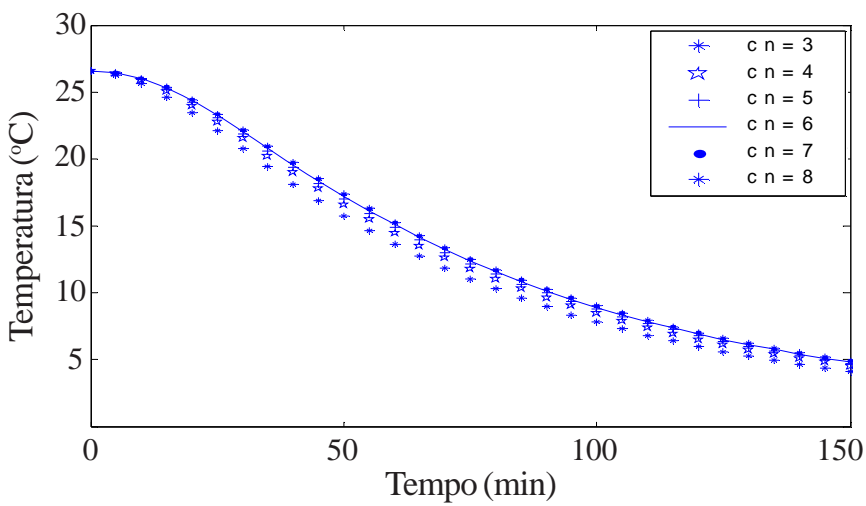

Figura 1. Curvas de resfriamento simuladas pelo uso do MDF, $\operatorname{com~}_{\mathrm{c}}=20 \mathrm{~W} \mathrm{~m}^{-2}{ }^{\circ} \mathrm{C}^{-1}$ e para valores de $\mathrm{cn}=3,4, \ldots 8$

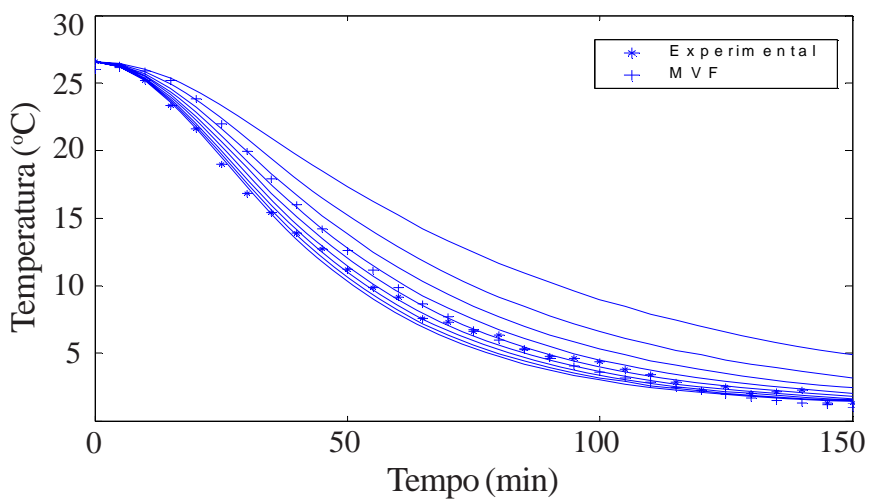

Figura 2. Curvas de resfriamento experimental, teórica pelo uso do MVF com $h_{c}=56,48 \mathrm{~W} \mathrm{~m}^{-2}{ }^{\circ} \mathrm{C}^{-1}$ e teóricas pelo uso do MDF para valores de $h_{c}=10,20, \ldots 100 \mathrm{~W} \mathrm{~m}^{-2}{ }^{\circ} \mathrm{C}^{-1}$

Após se observar os resultados mostrados na Figura 2, conclui-se que o valor de $h_{c}$ representativo do processo de resfriamento simulado pelo MDF está no intervalo $\mathrm{IHC}=[50$, 80]; sendo assim, uma conclusão efetiva só é possível a partir da análise dos resíduos, entre os resultados experimentais e os teóricos, para os vários valores de $h_{c}$ neste intervalo IHC. Na Tabela 1 tem-se os resíduos obtidos da comparação entre os resultados experimentais e os simulados pelo MDF, considerando-se $h_{c}=50,60, \ldots 100 \mathrm{~W} \mathrm{~m}^{-2}{ }^{\circ} \mathrm{C}^{-1}$.
Tabela 1. Resíduos obtidos da comparação entre os resultados experimentais e os teóricos em função do $h_{c}$ entre 50 e $100 \mathrm{~W} \mathrm{~m}^{-2}{ }^{\circ} \mathrm{C}^{-1}$

\begin{tabular}{cc}
$\mathrm{h}_{\mathrm{c}}\left(\mathrm{Wm}^{-2}{ }^{\circ} \mathrm{C}^{-1}\right)$ & Resíduos \\
\hline 50 & 6,2394 \\
60 & 3,9860 \\
70 & 3,0151 \\
80 & 3,1318 \\
90 & 3,7493 \\
100 & 4,4615 \\
\hline
\end{tabular}

Tabela 2. Resíduos obtidos da comparação entre os resultados experimentais e os teóricos em função do $h_{c}$ entre 60 e $80 \mathrm{~W} \mathrm{~m}^{-2}{ }^{\circ} \mathrm{C}^{-1}$

\begin{tabular}{cccc}
$\begin{array}{c}\text { Valor de } \mathrm{h}_{\mathrm{c}} \\
\left(\mathrm{Wm}^{-2} \mathrm{C}^{-1}\right)\end{array}$ & Resíduos & $\begin{array}{c}\text { Valor de } \mathrm{h}_{\mathrm{c}} \\
\left(\mathrm{Wm}^{-2} \mathrm{C}^{-1}\right)\end{array}$ & Resíduos \\
\hline 60 & 3,9860 & 71 & 2,9857 \\
61 & 3,8314 & 72 & 2,9673 \\
62 & 3,6897 & 73 & 2,9591 \\
63 & 3,5608 & 74 & 2,9606 \\
64 & 3,4448 & 75 & 2,9711 \\
65 & 3,3417 & 76 & 2,9897 \\
66 & 3,2514 & 77 & 3,0159 \\
67 & 3,1738 & 78 & 3,0487 \\
67 & 3,1088 & 79 & 3,0876 \\
69 & 3,0560 & 80 & 3,1318 \\
70 & 3,0151 & & \\
\hline
\end{tabular}

A partir da análise dos valores do resíduo como função dos valores de $h_{c}$ como mostrados na Tabela 1 , nota-se que 0 melhor valor de $h_{c}$ está entre 60 e $80 \mathrm{~W} \mathrm{~m}^{-20} \mathrm{C}^{-1}$. Para investigar com maior precisão, o melhor valor de $\mathrm{h}_{\mathrm{c}}$ neste intervalo, temse os resultados indicados na Tabela 2, do que se conclui que o melhor valor inteiro de $h_{c}$ é aquele próximo a $73 \mathrm{~W} \mathrm{~m}^{-2}{ }^{\circ} \mathrm{C}^{-1}$. Maior precisão pode ser alcançada continuando-se com o mesmo raciocínio. $\mathrm{O}$ valor de $\mathrm{h}_{\mathrm{c}}$ difere em aproximadamente 23\% daquele obtido por Teruel et al., (2001), que obtiveram $\mathrm{h}_{\mathrm{c}}=56,48 \mathrm{Wm}^{-20} \mathrm{C}^{-1}$, com desvio de cerca de $7,07 \%$ quando comparado com os dados experimentais. De acordo com o que consta em Lima (1999), esta diferença pode ser atribuída à

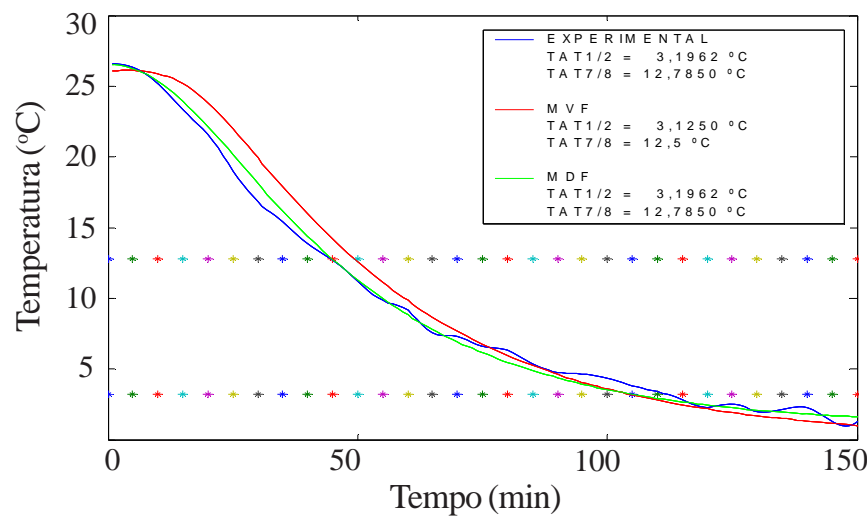

Figura 3. Ajustes por splines cúbicas dos dados experimentais e dos dados teóricos obtidos tanto pelo uso do MVF com $\mathrm{h}_{\mathrm{c}}=56,48 \mathrm{~W} \mathrm{~m}^{-2}{ }^{\circ} \mathrm{C}^{-1}$ quanto pelo uso do MDF com $\mathrm{h}_{\mathrm{c}}=73$ $\mathrm{W} \mathrm{m} \mathrm{m}^{-20} \mathrm{C}^{-1}$ 
dimensão do modelo, ao tamanho da malha espacial considerada entre outros fatores. Observar que no caso do uso do referido MVF usou-se uma malha de $20 \times 20$.

Na Figura 3 tem-se os ajustes por splines cúbicas para os dados experimentais e dos dados teóricos obtidos tanto pelo uso do MVF com $h_{c}=56,48 \mathrm{~W} \mathrm{~m}^{-2}{ }^{\circ} \mathrm{C}^{-1}$, quanto pelo MDF com $\mathrm{h}_{\mathrm{c}}=73 \mathrm{~W} \mathrm{~m}^{-2}{ }^{\circ} \mathrm{C}^{-1}$, e os respectivos valores de TAT para 1/2 e 7/ 8 (as linhas horizontais mostram os valores dos dados experimentais).

\section{CONCLUSÕES}

1. Analisando-se os resultados conclui-se pela adequada proximidade entre a curva experimental e a curva simulada pelo MDF com $h_{c}=73 \mathrm{~W} \mathrm{~m}^{-2}{ }^{\circ} \mathrm{C}^{-1}$, com desvio de cerca de 2,95\%, quando comparado com os dados experimentais, e a conseqüente concordância exata dos valores de TAT.

2. Sugere-se o uso do método numérico de diferenças finitas implícito, implementado no Matlab 6.1, para simular o processo do resfriamento de frutas esféricas.

3. Há necessidade da continuação de estudos para avaliar a sensibilidade do valor de $h_{c}$ para determinação efetiva de seu valor bem como de sua interpretação.

\section{LITERATURA CITADA}

Amendola, M. Analysis of the parameters associated to the numerical simulation of the heat transfer process in agricultural products. In: International Workshop on Information Technologies and Computing Techniques for Agro-Food Sector-Afot. Barcelona - Spain, 2003, 8p.

Amendola, M. Sistemas de suporte a decisão para processos agrícolas: simulação numérica de processos de transferência de calor. Mini-curso. Congresso Temático de Dinâmica e Controle-DINCON, da SBMAC. 3. Ilha Solteira - Brasil, 2004. 30p.

Amendola, M.; Teruel, B. Uso de un esquema implícito y de splines para la simulación numérica del proceso de enfriamiento de naranjas. In: Congreso Iberoamericano de Tecnología de Poscosecha y Agro exportaciones, 3, Santiago, 2002, p.141.

ASHRAE. American Society of Heating, Refrigerating and AirConditioning Engineers. Systems and applications. methods of precooling of fruits, vegetables and flowers. Atlanta, 1994. Chapter 10, p.10.1-10.10.

Benavides, M.N.; Avendaño, G.O. Curvas de congelación y descongelación de fresas y frambuesas calculadas con el método de diferencias finitas. In: Congreso Iberoamericano de Aire Acondicionado y Refrigeración, v.1, 1997. p.154159.

Boldrini, J.L.; Rodrigues Costa, S.I., Figueiredo, V.L.; Wetzler, H.G. Álgebra linear. 3ed. São Paulo: Harper \& Row no Brasil, 1980.372p.
Castro, L.R.; Amendola, M. Simulação numérica do processo de transferência de calor em vegetais esféricos. In: Congresso Ibero Latino - Americano em Métodos Computacionais e Engenharia, 20, São Paulo, 1999, CDRom

Chau, K.V.; Gaffney, J.J.; Baird, C.D.; Church, G.A. Resistance to air flow of oranges in bulk and in cartons. Transactions of ASAE, St. Joseph, v.28, n.6, p.2083-2088, 1985.

Cleland, D.J.; Earle, R.L. Freezing time prediction of foods-a simplified procedure. Revue Internationale du Froid, Paris, v. 5, n.3, p.134-140, 1982.

Cunha, C. Métodos numéricos. 2ed. Campinas: Editora da Unicamp, 2000. 265p.

Dincer, I. An effective method for analysing of precooling process parameters. International Journal of Energy Research, New York, v.19, p.95-102, 1995.

Fraser, H.; Otten, L. Predicting $7 / 8$ cooling times for peaches by comparing heat transfer modelling and field measurement methods. American Society of Agricultural Engineers, St. Joseph, Paper no. 92-6016. 10p. 1992.

Hayakawa, K. Computerized simulation for heat transfer and moisture loss from an idealized fresh produce. Transactions of the ASAE, St. Joseph, v.21, p.4-6, 1978.

Hayakawa, K.; Succar, J. Heat transfer and moisture loss of spherical fresh produce. Journal of Food Science, Chicago, v.47, p.596-605, 1982.

Incropera, F.P.; Witt, D.P. Fundamentals of heat and mass transfer. Rio de Janeiro: LTC, 1990. 455p.

Lima, A.G.B. Fenômeno de difusão em sólidos esferoidais prolatos. Estudo de caso: secagem de banana. Campinas: UNICAMP, 1999. 244p. Tese Doutorado

Mohsenin, N.N. Thermal properties of foods and agricultural materials. New York: Gordon and Breach, 1980. 405p.

Ramos, A.L.; Palmisano, E.; Dombey, A.; Pimentel, J.A.; Fayés, D.; González, D. Thermal properties of tropical fruits and vegetables. Revista Española de Ciencia e Tecnología de los Alimentos, Madrid, v.33, n.3, p.271-283, 1993.

Richtmyer, R.D.; Morton, K.W. Difference methods for initial value problems. New York: Interscience, 1967. 401p.

Ruggiero, M.A.G.; Lopes, V.L.R. Cálculo numérico: aspectos teóricos e computacionais. 2ed. São Paulo: Makron, 1996. 406p.

Smith, R. E., Bennet, A.H. Mass average temperature of fruits and vegetables during transient cooling. Transaction of ASAE, St. Joseph, v. 8, n. 2, p. 249, 1965.

Teruel, B.; Cortez, L.; Leal, P.; Lima, A. Estudo teórico do resfriamento com ar forçado de frutas de geometrias diferentes. Ciência e Tecnologia de Alimentos, Campinas, v.21, n.2, p.228-235, 2001

Trelea, I.C.; Alvarez, G.; Trystram, G. Nonlinear predictive optimal control of a batch refrigeration process. Journal of Food Process Engineering, Westport, v.21, p.1-32, 1998. 